\title{
A Novel Tri-Beam Antenna System Based on U-Shaped Dipole
}

\author{
Xi Wang DAI ${ }^{1,2}$, Guo Qing LUO ${ }^{1}$, Hua Yan JIN ${ }^{1}$, Zhen $L I A O^{1}$, Zhang Cheng HAO \\ ${ }^{1}$ Key Laboratory of RF Circuits \& System of Ministry of Education, Institute of Antennas and Microwave Technology, \\ Hangzhou Dianzi University, Hangzhou, 310018, China \\ ${ }^{2}$ State Key Laboratory of Millimeter Waves, School of Information Science and Engineering, \\ Southeast University, Nanjing, 210096, China
}

\{xwdai, luoguoqing, huayan_jin, liaozhen\}@hdu.edu.cn, zchao@seu.edu.cn

Submitted June 3, 2018 / Accepted October 27, 2018

\begin{abstract}
Consisting of 6 radiating elements and a $3 \times 3$ Butler matrix, a novel tri-beam antenna system is proposed in this paper. The radiating element consists of two U-shaped arms and coupled strips, which shows a wide impedance characteristic. Three branch line couplers and four $-90^{\circ}$ phase shifters are combined into $3 \times 3$ Butler matrix as a beam-forming network (BFN). In order to avoid the crossover of transmission lines, one main line of a $1.76 \mathrm{~dB}$ coupler is designed and located between two $3 \mathrm{~dB}$ couplers. With this arrangement, the signal from one input port can be divided into three output signals with equal amplitude and specified phase differences of $0^{\circ}$, $+120^{\circ}$ and $-120^{\circ}$. Furthermore, a $2 \times 3$ antenna array is connected with this BFN for three orthogonal beams. Measured results show that three beams at $\theta=0^{\circ}, 40^{\circ}$ and $-40^{\circ}$ are produced when different input ports are excited.
\end{abstract}

\section{Keywords}

U-shaped arm, Butler matrix, tri-beam antenna

\section{Introduction}

Multi-beam antenna can form several narrow beams to cover specific area for the signal transmission in wireless communication system [1-3]. Comparing with the single beam antenna, multiple-beam antenna can have a higher gain and a better signal to noise ratio, which leads to the increased coverage for rejecting interference from co-channel reuse cells in mobile communication system [4]. As one of well-known beam-forming network, Butler matrix is a passive element with $\mathrm{N}$ input ports and can divide an input signal into $\mathrm{N}$ output ports [5], [6]. The low power loss and the simplicity in structure of the Butler matrix guarantee its prevalence in design of multiple-beam antenna [7-12]. However, the number of input/output ports of conventional Butler matrix is equal to $2^{m}$ ( $m$ represents a positive integer) [8]. Therefore, the symmetrical beams formed by the Butler matrix are located on both sides of the

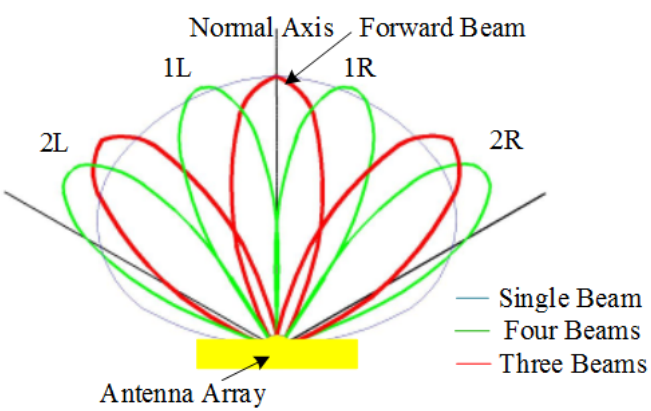

Fig. 1. Signal coverage with different-beam antenna.

antenna's normal axis, shown in Fig. 1. This restriction of a traditional Butler matrix greatly limits the flexibility of the design and leads to a high cost.

In order to improve the signal transmission and reduce mutual interference, a forward beam along the antenna's normal axis is preferred to be created. With the same signal coverage area, multi-beam antenna can have larger system capacity and better signal level, shown in Fig. 1. Several methods have been proposed to improve the Butler matrix for better signal coverage [10-16]. Without using any crossovers, a $4 \times 4$ Butler matrix with broadband characteristic and CPW technology is proposed in [13]. As a key component, two-layer slot-coupled directional coupler is adopted to reduce the circuit size and the loss. A compact Butler matrix without phase shifter is introduced in [14]. By employing planar couplers with different phase differences, the Butler matrix avoids the interconnecting mismatch loss and imbalanced amplitude. Thus the circuit has a wide working frequency band of $20.1 \%$ and a small imbalance less than $0.4 \mathrm{~dB}$. An asymmetric Butler matrix with 3 input ports and 4 output ports is proposed in [15]. With Wilkinson divider, the asymmetric structure allows to create a forward beam along the array's normal axis. In order to suppress spurious frequencies and provide near-band rejection, a novel Butler matrix with inherent bandpass filter transfer functions is presented in [16]. The new circuit has advantages of size and mass reduction due to its coupled resonators with characteristics of the power division, phase distribution, and filtering transfer function. 
In [17], the authors analyzed different multibeam network with odd number of inputs or non-orthogonal pattern. Three-way directional coupler for orthogonal three-beam antenna array is proposed in [18]. However, dual microstrip layer are needed for the cross connect, which increase the cost and reduce design flexibility. Based on the SIW and multifolded structure, a $4 \times 8$ Butler matrix with working frequency of $38 \mathrm{GHz}$ is designed for $5 \mathrm{G}$ applications [19]. A low-complexity beam-switching array using a standard $4 \times 4$ Butler matrix and the phase reconfigurable synthesized transmission line (PRSTL) is demonstrated in [20]. With the help of 1-bit phase shifter function of PRSTL, the array has enhanced beam controllability and widened spatial coverage.

In this paper, a novel tri-beam antenna system is proposed for wireless communication application. The element of $2 \times 3$ antenna array is designed with two U-shaped arms and coupled patch for broadband operation. The Butler matrix is composited of three branch line couplers and four $-90^{\circ}$ phase shifters. With the help of one non equilibrated coupler, the signals at three output ports can have the equal amplitude with accurate phase difference of $0^{\circ},+120^{\circ}$ and $-120^{\circ}$. By connecting this circuit with the antenna array, three beams including one forward beam along the antenna's normal axis are obtained. It can split original 65 degree coverage into three sectors with separate narrow beams, which can reduce the overlap interference and improve efficient spectrum reuse. It has a potential application in the mobile communication systems.

\section{Theoretical Analyses}

\subsection{Radiating Element}

The modified dipole unit is chosen as the radiating element for tri-beam antenna system due to its broadband impedance characteristic and high gain performance. The element consists of two U-shaped arms and coupled strips, which shows a wide impedance bandwidth and vertical polarization characteristics. An integrated balun with $\lambda / 4$ height is located between U-shaped arms and metal reflector, which is shorted at the metal reflector and provides a balanced feed to the two U-shaped arms. In order to broad the working frequency band and improve the front to back ratio, several coupled strips printed on FR4 substrate are utilized. The geometry of radiating element is given in Fig. 2, and its main parameters are summarized in Tab. 1.

The antenna design procedures can be summarized as follows: Firstly, according to the resonant frequency, the height of dipole $H_{\mathrm{d}}$ can be determined, $H_{\mathrm{d}}=\lambda_{0} / 4$ Then the dimensions of U-shaped arms can be calculated. The length $L_{\mathrm{d}}$ is related with the resonant frequency, while the width $W_{\mathrm{d}}$ is related with the working frequency band. Next the triangular patch should be optimized due to its huge impact on the matching. Lastly, several coupled strips are added to improve its bandwidth and radiation performance.

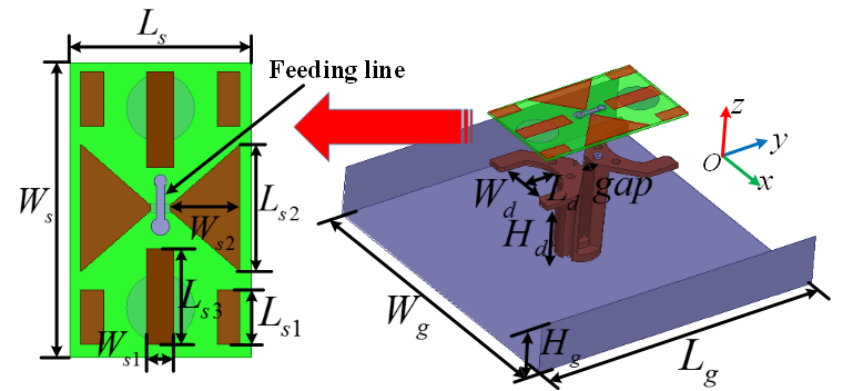

Fig. 2. Geometry of the proposed radiating element.

\begin{tabular}{|c|c|c|c|}
\hline Parameter & Value (mm) & Parameter & Value (mm) \\
\hline$W_{g}$ & 120 & $L_{g}$ & 120 \\
\hline$W_{s}$ & 36 & $L_{s}$ & 62 \\
\hline$W_{s 1}$ & 5 & $L_{s 1}$ & 11 \\
\hline$W_{s 2}$ & 15 & $L_{s 2}$ & 28 \\
\hline$W_{d}$ & 22.4 & $L_{d}$ & 16 \\
\hline$H_{g}$ & 20 & $L_{s 3}$ & 21 \\
\hline$H_{d}$ & 34 & gap & 5 \\
\hline
\end{tabular}

Tab. 1. Parameters of the radiating element.

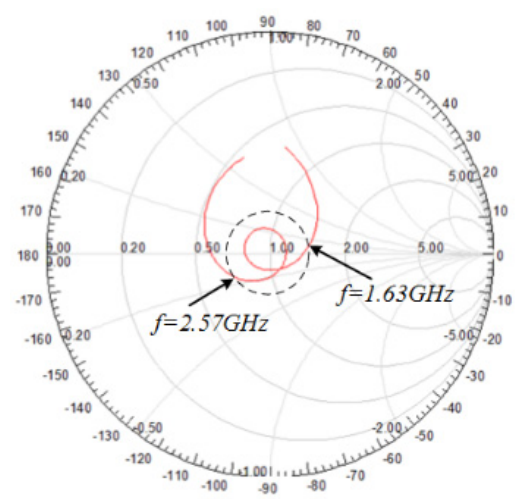

(a)

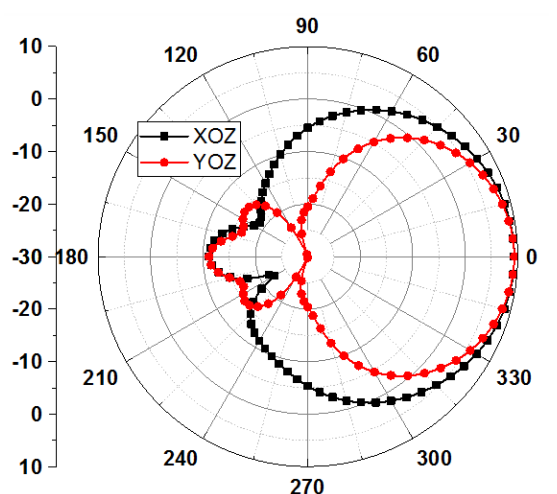

(b)

Fig. 3. Performance of the radiating element. (a) Smith chart, (b) radiation pattern.

The performance of radiating element is analyzed with EM software of ANSYS HFSS v15 and its performance is shown in Fig. 3. It can be seen that the return loss is lower than $-15 \mathrm{~dB}$ at $1.63 \sim 2.57 \mathrm{GHz}$, and the front to back ratios of XOZ- and YOZ-plane radiation patterns are better than $20 \mathrm{~dB}$ at the center frequency. The gain of the 


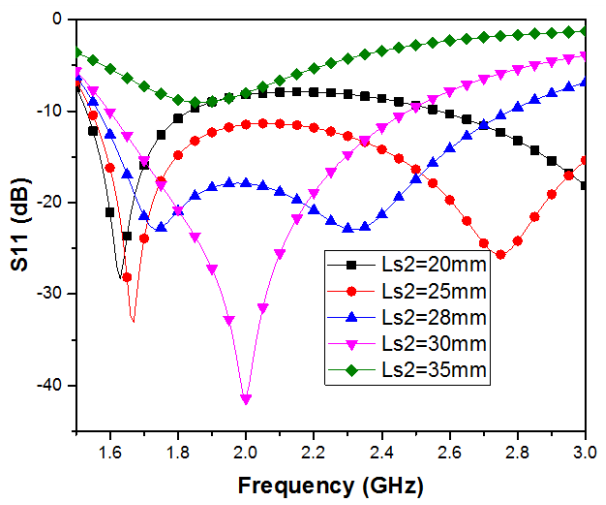

Fig. 4. Reflection coefficient varied with parameter $L_{\mathrm{s} 2}$.

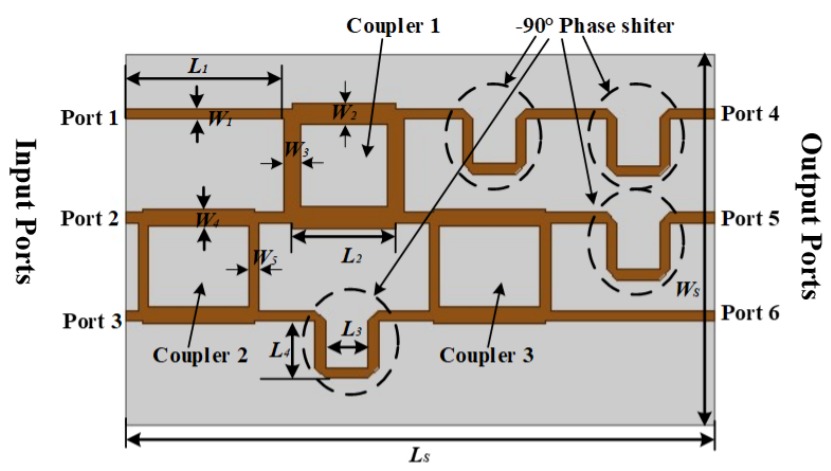

Fig. 5. Geometry of the proposed Butler matrix.

radiating element is varied from 8 to $10 \mathrm{dBi}$, while the half power bandwidth (HPBW) of XOZ-plane are round $65^{\circ}$ over the whole working frequency band. In order to analyze the mechanism of radiating element, a parameter study is carried out. While the parameter $L_{\mathrm{s} 2}$, length of triangular coupled patch, varied from $20 \mathrm{~mm}$ to $40 \mathrm{~mm}$, the reflection coefficient $\left(S_{11}\right)$ of the radiating element is shown in Fig. 4. It can be observed that the coupling patch has a great influence on the reflection coefficient. The value of $L_{\mathrm{s} 2}$ is chosen as $28 \mathrm{~mm}$ for the appropriate bandwidth and good return loss.

\section{$2.23 \times 3$ Butler Matrix}

The geometry of the proposed $3 \times 3$ Butler matrix is shown in Fig. 5. Three branch line couplers and four $-90^{\circ}$ phase shifters consist the circuit. Coupler 1 is $1.74 \mathrm{~dB}$ coupler with $90^{\circ}$ phase difference between two output ports, while Coupler 2 and Coupler 3 are $3 \mathrm{~dB}$ couplers. The $-90^{\circ}$ phase shifter is formed by the meander line for its compact structure. When the signal is excited at port 1 , the signals at three output ports with equal amplitude and a phase difference of $0^{\circ}$ are obtained. A forward beam along the antenna normal axis can be formed when the signal is excited at port 1 . Similar analysis can be made when port 2 and port 3 are excited, respectively. The signals at three output ports are shown in Fig. 6. With the help of one non equilibrated coupler, the signals at three output ports can have the equal amplitude with accurate phase difference of $-120^{\circ}$ and $+120^{\circ}$. Thus, a right $/$ left tilted beam can be produced when port 2 or port 3 is connected with antenna elements, respectively.

Assuming that the circuit has perfect matching, infinite isolation and no loss, the scattering parameters of the proposed Butler matrix can be written as follows:

$$
\mathbf{S}=\frac{1}{\sqrt{3}}\left[\begin{array}{cccccc}
0 & 0 & 0 & \mathrm{e}^{\mathrm{j} 0} & \mathrm{e}^{\mathrm{j} 0} & \mathrm{e}^{\mathrm{j} 0} \\
0 & 0 & 0 & \mathrm{e}^{\mathrm{j} \frac{\pi}{2}} & \mathrm{e}^{-\mathrm{j} \frac{\pi}{6}} & \mathrm{e}^{-\mathrm{j} \frac{5 \pi}{6}} \\
0 & 0 & 0 & \mathrm{e}^{\mathrm{j} 0} & \mathrm{e}^{\mathrm{j} \frac{2 \pi}{3}} & \mathrm{e}^{-\mathrm{j} \frac{2 \pi}{3}} \\
\mathrm{e}^{\mathrm{j} 0} & \mathrm{e}^{\mathrm{j} \frac{\pi}{2}} & \mathrm{e}^{\mathrm{j} 0} & 0 & 0 & 0 \\
\mathrm{e}^{\mathrm{j} 0} & \mathrm{e}^{-\mathrm{j} \frac{\pi}{6}} & \mathrm{e}^{\mathrm{j} \frac{2 \pi}{3}} & 0 & 0 & 0 \\
\mathrm{e}^{\mathrm{j} 0} & \mathrm{e}^{-\mathrm{j} \frac{5 \pi}{6}} & \mathrm{e}^{-\mathrm{j} \frac{2 \pi}{3}} & 0 & 0 & 0
\end{array}\right] .
$$

According to the above analysis, the proposed Butler matrix is designed and simulated at $2.0 \mathrm{GHz}$, whose geometry is depicted in Fig. 5. The circuit is designed on AD300 substrate with a dielectric constant of 3.0, a thickness of $0.8 \mathrm{~mm}$ and a loss tangent of 0.002 . The branch lines of Coupler 2 and Coupler 3 have the same dimensions, a width of $2.75 \mathrm{~mm}$ and a length of $28 \mathrm{~mm}$, while the main line has a width of $4.4 \mathrm{~mm}$ and the same length. Coupler 1 is an unequal coupler with different widths for branch line and main line. Four phase shifters are the same with its $2.75 \mathrm{~mm}$ width and $31 \mathrm{~mm}$ length. The geometric parameters for the tri-beam Butler matrix are listed in Tab. 2. The structure is simulated by using ANSYS HFSS software based on the finite element method.

With the characteristic of branch line coupler, high isolation between input ports is achieved for this structure. Four phase shift of $-90^{\circ}$ is designed by using a meander transmission line section. Even this structure is asymmetric,

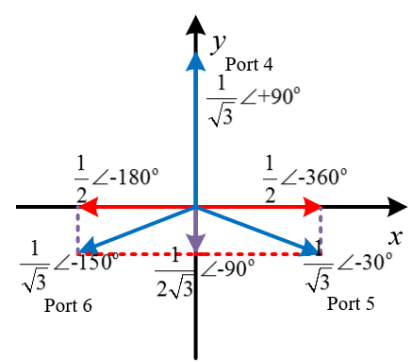

(a)

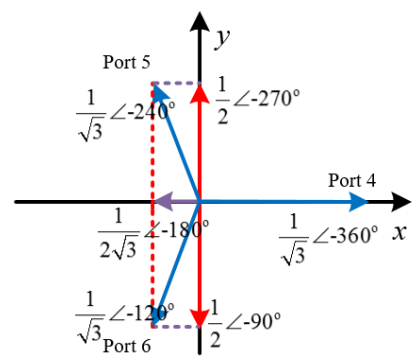

(b)
Fig. 6. Output signals at Port 4/5/6 when different ports are excited: (a) Port 2, (b) Port 3.

\begin{tabular}{|c|c|c|c|}
\hline Parameter & Value (mm) & Parameter & Value (mm) \\
\hline$W_{\mathrm{s}}$ & 120 & $L_{\mathrm{s}}$ & 120 \\
\hline$W_{1}$ & 36 & $L_{1}$ & 62 \\
\hline$W_{2}$ & 5 & $L_{2}$ & 11 \\
\hline$W_{3}$ & 15 & $L_{3}$ & 28 \\
\hline$W_{4}$ & 22.4 & $L_{3}$ & 16 \\
\hline$W_{5}$ & 20 & & \\
\hline
\end{tabular}

Tab. 2. Parameters of the proposed Butler matrix. 


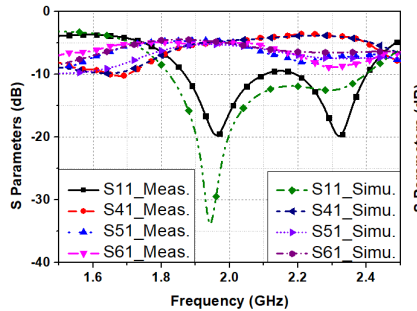

(a)

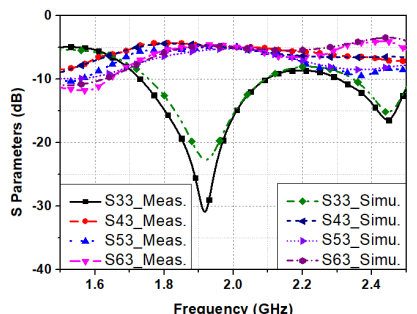

(c)

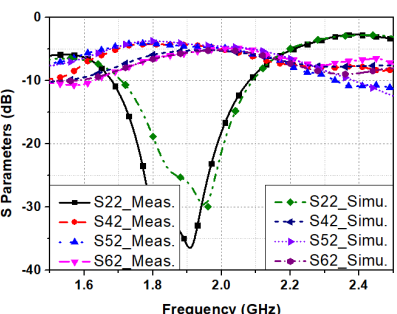

(b)

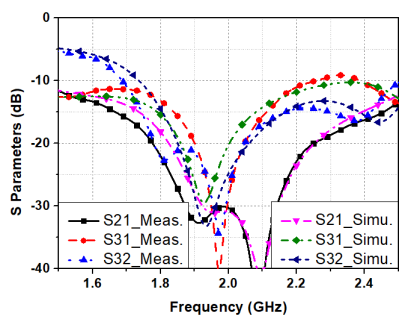

(d)
Fig. 7. Measured and simulated $S$ parameters of the proposed $3 \times 3$ Butler matrix when different ports are excited. (a) Port 1, (b) Port 2, (c) Port 3, (d) Isolation.

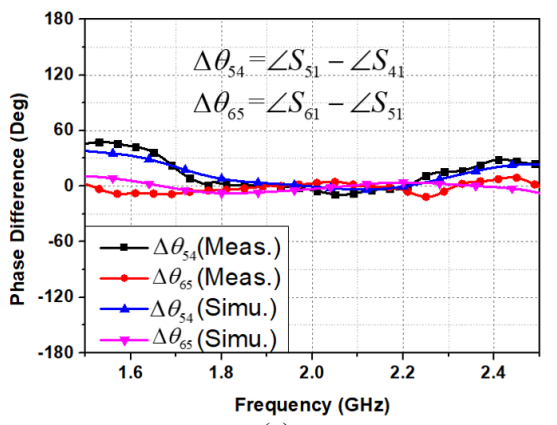

(a)

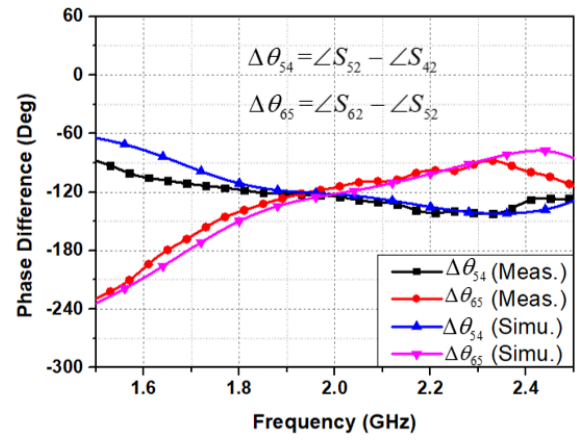

(b)

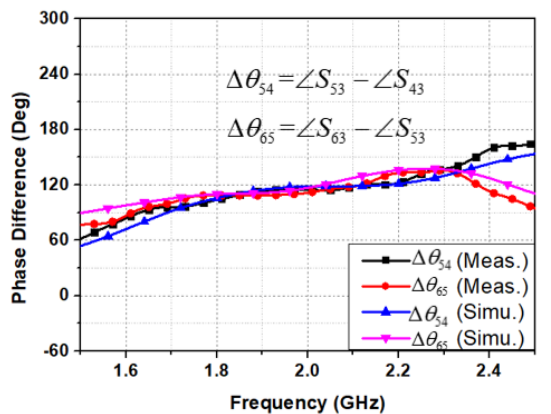

(c)

Fig. 8. Phase differences between two adjacent outputs when different input ports are excited: (a) Port 1, (b) Port 2, (c) Port 3. the input signal from port $1 / 2 / 3$ can be divided into three output ports with equal amplitude and specific phase difference.

Figure 7 shows the simulated reflection and transmission characteristic of the proposed structure when different input port is excited and other ports are matched. It can be noticed that the transmission level for three output ports is around $-4.9 \mathrm{~dB}$ with a difference less than $0.5 \mathrm{~dB}$ at the working frequency of $2.0 \mathrm{GHz}$, meanwhile, the reflections of different input ports are less than $-15 \mathrm{~dB}$.

Phase difference of adjacent output ports is an important factor for the Butler matrix. We define it in the following form,

$$
\begin{aligned}
& \Delta \theta_{54}=\angle S_{5 i}-\angle S_{4 i}, \\
& \Delta \theta_{65}=\angle S_{6 i}-\angle S_{5 i}
\end{aligned}
$$

where $i$ represents the input port 1, 2, 3, respectively. The simulated phase differences of the proposed circuit are illustrated in Fig. 8. It can be seen that phase differences of $0^{\circ},-120^{\circ}$ and $+120^{\circ}$ are obtained when different input ports are excited, respectively. According to (4), beam scanning equation of planar antenna array, we can calculate the directions of beam with the phase differences. The phase differences on the output ports can produce three beams with the maximum radiation values in the directions of $0^{\circ},+42^{\circ}$ and $-42^{\circ}$, respectively.

$$
\theta_{\mathrm{d}}=\arcsin \left(\frac{\lambda}{2 \pi d} \Delta \theta_{i j}\right)
$$

In order to validate the performance of the proposed structure, the equivalent circuit with ideal elements is given in Fig. 9(a). It can be noticed that two $-90^{\circ}$ delay elements

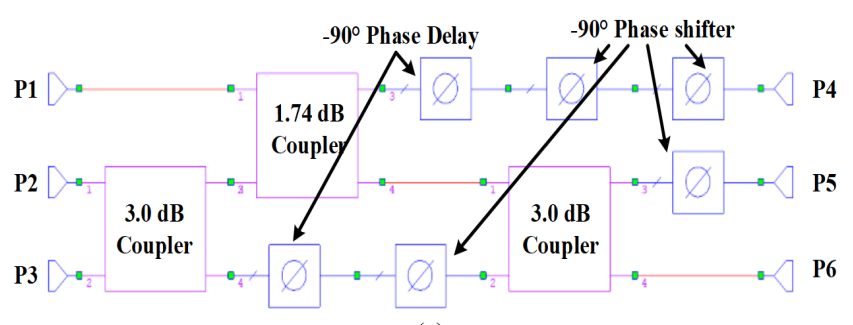

(a)

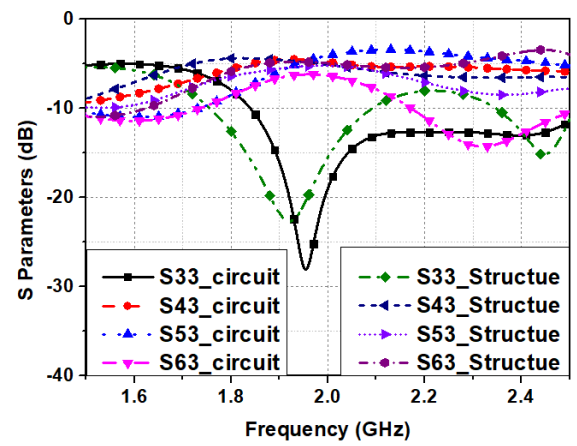

(b)

Fig. 9. The equivalent circuit and its performance. (a) The circuit, (b) frequency responses. 


\begin{tabular}{|c|c|c|c|c|c|}
\hline Ref. & Ports & Structure & $\begin{array}{c}\text { Center } \\
\text { frequency } \\
(\mathbf{G H z})\end{array}$ & $\begin{array}{c}\text { Amplitude } \\
\text { imbalance } \\
(\mathbf{d B})\end{array}$ & $\begin{array}{c}\text { Phase } \\
\text { error } \\
\text { (degree) }\end{array}$ \\
\hline$[3]$ & $4 \times 4$ & $\begin{array}{c}\text { Lumped } \\
\text { element }\end{array}$ & 1.0 & 1.5 & 10 \\
\hline$[5]$ & $4 \times 4$ & SIW & 9.5 & 0.6 & 5 \\
\hline$[9]$ & $4 \times 4$ & Slot line & 5.8 & 3 & - \\
\hline$[15]$ & $3 \times 4$ & One layer & 2.4 & 1.5 & 2.21 \\
\hline $\begin{array}{c}\text { Our } \\
\text { work }\end{array}$ & $3 \times 3$ & One layer & 2.0 & 0.5 & 2 \\
\hline
\end{tabular}

Tab. 3. Comparison of the performance of the proposed Butler matrix with other Butler matrices.

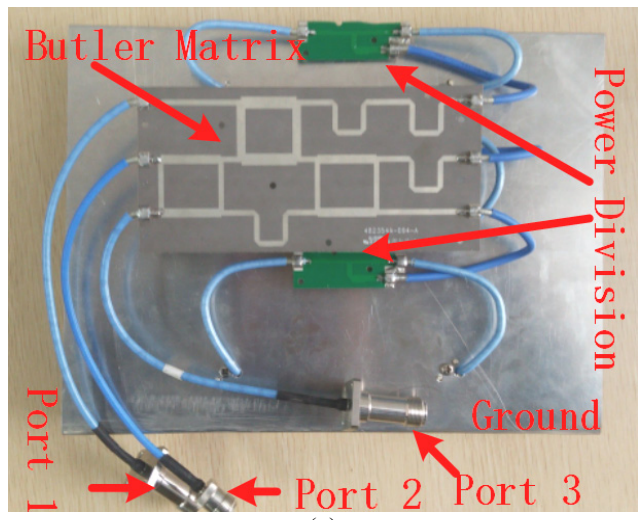

(a)

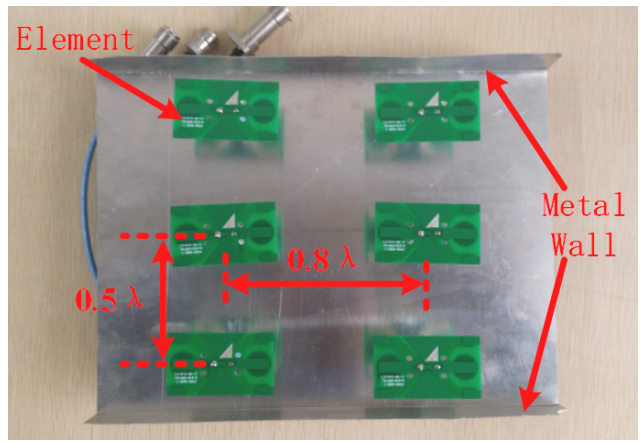

(b)

Fig. 10. Photograph of the tri-beam antenna array. (a) Back view, (b) top view.

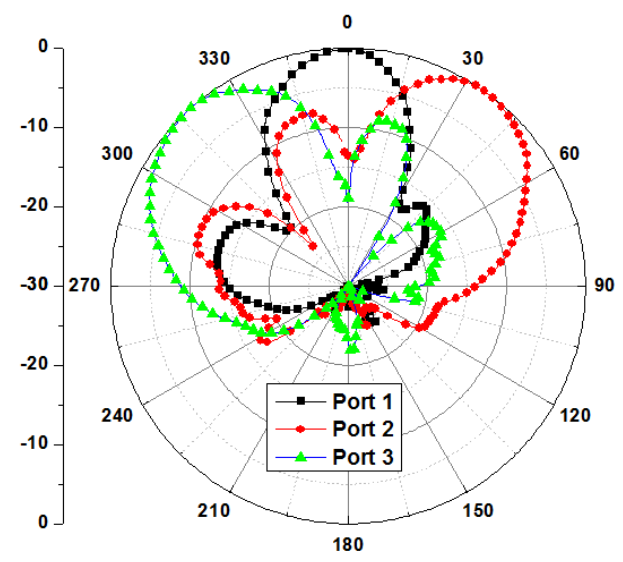

Fig. 11. Measured radiation patterns of the proposed tri-beam antenna array. are added in the circuit, which are used to be equivalent to the phase delay induced by the couplers. The frequency responses of the circuit and the structure are given in Fig. 9(b). Only the performance of port 3 is given for the similarity of other two ports. It can be observed that the return loss of the circuit is better than that of the structure. The reason is good matching of the ideal elements in the circuit. In the whole, good agreement is achieved between the circuit and the structure.

Several recently published Butler matrices are selected to be compared with the proposed Butler matrix, which is given in Tab. 3. The proposed one can be designed with one layer substrate, and has a minimum amplitude imbalance and phase error. When connecting with the antenna array, the proposed Butler matrix can create three beams with one forward beam along the normal axis.

\section{Experiment Results}

As a tri-beam forming network, the proposed Butler matrix is connected to a $2 \times 3$ antenna array, shown in Fig. 10. Each row has two dipole elements spaced by $0.8 \lambda_{0}$ at $2.0 \mathrm{GHz}$, and connected with equal power divider. In order to reduce the mutual coupling between elements, the distance between adjacent rows is designed to be $0.5 \lambda_{0}$, which also guarantees the orthogonality between different beams. The antenna has a resonant frequency at $2.0 \mathrm{GHz}$ with return loss less than $-20 \mathrm{~dB}$. The minimum mutual coupling between elements is achieved due to its spacing, and the isolation is better than $25 \mathrm{~dB}$.

Figure 11 shows the measured radiation patterns of this antenna array with the Butler matrix. It can be observed that three beams at $\theta=0^{\circ}, 40^{\circ},-40^{\circ}$ are produced. When port 1 is excited, equal amplitude and $0^{\circ}$ phase difference at three output ports create a forward beam along the antenna's normal axis. Similarly, a beam tilt to right/left direction can be created when port $2 / 3$ is excited. The gains of three beams are $14.1,13.2$ and $13.3 \mathrm{dBi}$ at $2.0 \mathrm{GHz}$, respectively.

\section{Conclusions}

A novel antenna array with three orthogonal beams is proposed for wireless communication system. This system is composed of broadband radiating elements and $3 \times 3$ Butler matrix. Consisting of two U-shaped arms and several coupled strips, the radiating element has a broad impedance bandwidth characteristic. The proposed Butler matrix is consisted of three couplers and four $-90^{\circ}$ phase shifters, which provides three output ports with equal amplitude and specific phase difference. A tri-beam antenna array is designed with this structure, resulting in three orthogonal beams at $0^{\circ}, 40^{\circ}$ and $-40^{\circ}$. The proposed tri-beam antenna system has a good potential application for wireless communication. 


\section{Acknowledgments}

This work is supported partly by Public Projects of Zhejiang Province under Grant No. 2017C31068, partly by the Natural Science Foundation of China under Grant No. 61722107 and partly by the Key Laboratory of Millimeter Wave program under Grant No. K201810.

\section{References}

[1] LIAN, J. W., BAN, Y. L., XIAO, C., et al. Compact substrate integrated $4 \times 8$ Butler matrix with sidelobe suppression for millimeter-wave multibeam application. IEEE Antennas and Wireless Propagation Letters, 2018, vol. 17, p. 928-932. DOI: 10.1109/LAWP.2018.2825367

[2] KAWDUngta, S., JAIBANAUEM, P., PONGGA, R., et al. Superstrate-integrated switchable beam rectangular microstrip antenna for gain enhancement. Radioengineering, 2017, vol. 26, no. 2, p. 430-437. DOI: $10.13164 /$ re. 2017.0430

[3] GANDINI, E., ETTORRE, M., SAULEAU, R., et al. A lumpedelement unit cell for beam-forming networks and its application to a miniaturized Butler matrix. IEEE Transactions on Microwave Theory and Techniques, 2013, vol. 61, no. 4, p. 1477-1487. DOI: 10.1109/TMTT.2013.2248744

[4] HO, M. J., STUBER, G. L., AUSTIN, M. D. Performance of switched-beam smart antennas for cellular radio systems. IEEE Transactions on Vehicular Technology, 1998, vol. 47, no. 1, p. 10-19. DOI: $10.1109 / 25.661027$

[5] KARAMZADEH, S., RAFII, V., KARTAL, M., et al. Compact and broadband $4 \times 4$ SIW Butler matrix with phase and magnitude error reduction. IEEE Microwave and Wireless Components Letters, 2015, vol. 25, no. 12, p. 772-774. DOI: 10.1109/LMWC.2015.2496785

[6] BUTLER, J., LOWE, R. Beam-forming matrix simplifies design of electronically scanned antennas. Electronic Design, 1961, vol. 9, p. 170-173.

[7] ZHENG, S., CHAN, W. S. Compact Butler matrix using size reduced elements. Microwave and Optical Technology Letters, 2007, vol. 49, no. 7, p. 1519-1521. DOI: 10.1002/mop.22489

[8] DE LILLO, R. A. A high performance 8-input, 8-output Butler matrix beamforming network for ultra-broadband applications. In Proceedings of IEEE Antennas and Propagation Society International Symposium. Ann Arbor (MI, USA), 1993, vol. 1, p. 474-477. DOI: 10.1109/APS.1993.385304

[9] DENIDNI, T. A., NEDIL, M. Experimental investigation of a new Butler matrix using slotline technology for beamforming antenna arrays. IET Microwave, Antennas and Propagation, 2008, vol. 2, p. 641-649. DOI: 10.1049/iet-map:20060199

[10] ZHONG, L. H., BAN, Y. L., LIAN, J. W., et al. Miniaturized SIW multibeam antenna array fed by dual-layer $8 \times 8$ Butler matrix. IEEE Antennas and Wireless Propagation Letters, 2017, vol. 16, p. 3018-3021. DOI: 10.1109/LAWP.2017.2758373

[11] LUO, G. Q., DAI, X. W., SUN, W., et al. Design of tri-beam antenna systems. In IEEE International Conference on Microwave and Millimeter Wave Technology. Being (China), 2016, p. 2-5. DOI: 10.1109/ICMMT.2016.7761659

[12] SUN, W., DAI, X. W., LUO, G. Q., et al. Design of X-band antenna system with three beams. In IEEE International Workshop on Electromagnetics: Applications and Student Innovation Competition. 2016, p. 1-2. DOI: 10.1109/iWEM.2016.7504970
[13] NEDIL, M., DENIDNI, T. A., TALBI, L. Novel Butler matrix using CPW multilayer technology. IEEE Transactions on Microwave Theory and Techniques, 2006, vol. 54, no. 1, p. 499-507. DOI: 10.1109/TMTT.2005.860490

[14] TIAN, G., YANG, J. P., WU, W. A novel compact Butler matrix without phase shifter. IEEE Microwave and Wireless Components Letters, 2014, vol. 24, no. 5, p. 306-308. DOI: 10.1109/LMWC.2014.2306898

[15] NACHOUANE, H., NAJID, A., TRIBAK, A., et al. Wideband $3 \times 4$ Butler matrix using Wilkinson divider for MIMO applications. In Proceedings of the 5th International Conference on Next Generation Network \&Services. Casablanca (Morocco), 2014, p. 101-105. DOI: 10.1109/NGNS.2014.6990236

[16] TORNIELLI DI CRESTVOLANT, V., IGLESIAS, P. M., LANCASTER, M. J. Advanced Butler matrices with integrated bandpass filter functions. IEEE Transactions on Microwave Theory and Techniques, 2015, vol. 63, no. 10, p. 3433-3444. DOI: 10.1109/TMTT.2015.2460739

[17] GARCÍA-GASCO TRUJILlO, J., SIERRA PÉREZ, M., NOVO GARCÍA, A., et al. $3 \times 3$ multibeam network for a triangular array of three radiating elements: design and measurement. In Proceedings of EUROCON 2011 - International Conference on Computer as a Tool. Lisbon (Portugal), 2011, p. 1-4. DOI: 10.1109/EUROCON.2011.5929412

[18] DING, K. J., FANG, X. X., WANG, Y., et al. Printed dual-layer three-way directional coupler utilized as $3 \times 3$ beamforming network for orthogonal three-beam antenna array. IEEE Antennas and Wireless Propagation Letters, 2014, vol. 13, p. 911-914. DOI: 10.1109/LAWP.2014.2321971

[19] CAO, Y., CHIN, K. S., CHE, W., et al. A compact 38-GHz multibeam antenna array with multi-folded Butler matrix for $5 \mathrm{G}$ applications. IEEE Antennas and Wireless Propagation Letters, 2017, vol. 16, p. 2996-2999. DOI: 10.1109/LAWP.2017.2757045

[20] CHU, H. N., MA, T. G. An extended $4 \times 4$ Butler matrix with enhanced beam controllability and widened spatial coverage. IEEE Transactions on Microwave Theory and Techniques, 2018, vol. 66, no. 3, p. 1301-1311. DOI: 10.1109/TMTT.2017.2772815

\section{About the Authors ...}

Xi Wang DAI was born in Caoxian, Shandong, China. He received the B.S. and M.S. degrees in Electronic Engineering from Xidian University, Xi'an, Shaanxi, China, in 2005 and 2008, and his Ph.D degree in Electromagnetic Fields and Microwave Technology from Xidian University in 2014. From March 2008 to August 2011, he worked at Guangdong Huisu Corporation as a manager of antenna department. Now, he is working at the Hangzhou Dianzi University, Hangzhou, China. His current research interests involve metamaterials, omni-directional antenna, MIMO antenna and low-profile antenna.

Guo Qing LUO (corresponding author) received the B.S. degree from the China University of Geosciences, Wuhan, China, in 2000, the M.S. degree from the Northwest Polytechnical University, Xi'an, China, in 2003, and the Ph.D. degree from the Southeast University, Nanjing, China, in 2007. Since 2007 , he has been a Lecturer with the faculty of the School of Electronics and Information, Hangzhou Dianzi University, Hangzhou, China, and was promoted to Professor in 2011. From Oct. 2013 to Oct. 2014, he joined 
the Dept. of Electrical, Electronic and Computer Engineering, Heriot-Watt University, Edinburgh, U.K., as a Research Associate, where he was involved in developing low profile antennas for UAV applications. He has authored or co-authored over 70 technical papers in refereed journals and conferences and holds 16 patents. His current research interests include RF, microwave and mm-wave passive devices, antennas, and frequency selective surfaces. Dr. Luo was a recipient of the CST University Publication Award in 2007, the National Excellent Doctoral Dissertation of China in 2009, and the National Natural Science Award (the second class) of China in 2016. He is a member of the IEEE Antennas and Propagation Society and the Microwave Theory and Techniques Society. He has served as a reviewer for many technical journals, including the IEEE Trans. Antennas Propag., IEEE Trans. Microw. Theory Tech., IEEE Antennas Wireless Propag. Lett., and IEEE Microw. Wireless Comp. Lett. etc.

Hua Yan JIN was born in Hangzhou, Zhejiang Province, China, in 1989. She received the B.S. degree in Electronic Engineering and Ph.D. degree in Electromagnetic Field and Microwave Technology from the Nanjing University of Science and Technology (NUST), Nanjing, China, in 2011 and 2017, respectively. From August 2012 to January 2013, May 2013 to October 2013, and April 2014 to October 2014, she was an exchange student with the Chang Gung University, Taoyuan, Taiwan. She is currently a Lecturer with the School of Electronics and Information, Hangzhou Dianzi University. Her main research interests include millimeter-wave antennas, differential-fed antennas, filtering antennas and wideband microstrip antennas.

Zhen LIAO received the B.S. degree from the Nanchang Hangkong University, Nanchang, China, in 2005, the M.S. degree from the Nanjing University of Science and Technology (NUST), Nanjing, China, in 2012 and the Ph.D. degree from the Southeast University, Nanjing, China, in 2017. He is currently a Lecturer with the School of Electronics and Information, Hangzhou Dianzi University, Hangzhou, China. His current research interests include microwave plasmonics, antennas, and metamaterials.

Zhang Cheng HAO received the B.S. degree in Microwave Engineering from Xidian University, Xi'an, China, in 1997, and the M.S. and Ph.D. degrees in Radio Engineering from the Southeast University, Nanjing, China, in 2002 and 2006, respectively. In 2006, he was a Post-Doctoral Researcher with the Laboratory of Electronics and Systems for Telecommunications, École Nationale Supérieure des Télécommunications de Bretagne, Bretagne, France, where he was involved with developing millimeter-wave antennas. In 2007, he joined the Dept. of Electrical, Electronic and Computer Engineering, Heriot-Watt University, Edinburgh, U.K., as a Research Associate, where he was involved with developing multilayer integrated circuits and ultra-wide-band components. In 2011, he joined the School of Information Science and Engineering, Southeast University, Nanjing, as a Professor. He holds 20 granted patents and has authored and co-authored over 150 referred journal and conference papers. His current research interests involve microwave and millimeter-wave systems, submillimeter-wave and terahertz components and passive circuits, including filters, antenna arrays, couplers, and multiplexers. Dr. Hao has served as the reviewer for many technique journals, including IEEE Transactions on Microwave Theory and Techniques, IEEE Transactions on Antennas and Propagation, IEEE Antennas and Wireless Propagation Letters, and IEEE Microwave and Wireless Components Letters. He was a recipient of the Thousands of Young Talents presented by China government in 2011 and the High Level Innovative and Entrepreneurial Talent presented by Jiangsu, China, in 2012. 